\title{
Ultrasound and MRI features of penile augmentation by "Jamaica Oil" injection. A case series.
}

\author{
Joe Francis', Angeline Poh Choo Choo', Gervais Wansaicheong Khin-Lin² \\ ${ }^{1}$ Department of Diagnostic Radiology, Changi General Hospital, ${ }^{2}$ Department of Diagnostic Radiology, Tan Tock Seng \\ Hospital, Singapore
}

\begin{abstract}
Penile paraffinoma is a rare but well-known entity. There have been few studies describing the ultrasound and MRI features of penile paraffinoma. In this case series we describe the imaging findings of 4 patients who self-injected "Jamaica oil" into their penises for the purpose of penile augmentation and discuss the utility of US and MRI in the management of these patients.
\end{abstract}

Keywords: penis, ultrasonography, magnetic resonance imaging.

\section{Introduction}

Penile paraffinoma is a rare but a well-known entity with many cases reported from Asia, Russia and Eastern Europe [1,2]. Paraffinoma, also known as oleo granuloma and sclerosing lipogranulomatosis results from injection of foreign substance containing straight-chain hydrocarbons, such as paraffin or mineral oil [3]. The first case of mineral oil injected into genitalia was reported by Robert Gersuny in 1899 [4,5]. Injection of various other substances such as petroleum jelly, metallic mercury and even implantation of stone into the penis has been reported $[6,7]$. In our local context, one of the recently encountered non-medical methods of penile augmentation is self-injection of unknown mineral oil such as "Jamaica Oil". Motivation to undergo penile augmentation ranges from desire to increase penile girth or length to a perceived sense of sexual pleasure [8]. While there have

Received 08.08.2014 Accepted 09.09.2014

Med Ultrason

2014, Vol. 16, No 4, 372-376

Corresponding author: Dr Joe Francis

Department of Diagnostic Radiology,

Changi General Hospital

2 Simei Street 3, Singapore 529889

Phone: +65-96796677;

Fax: 65-62601703

Email: Joe_Francis@cgh.com.sg;

joefrancis01@gmail.com been several reports in the literature regarding foreign body granulomatous reaction in the penis, to our knowledge, there have been no studies describing the ultrasound (US) findings and few studies describing the MRI features in these patients. We describe the imaging findings in four patients who were seen in our institution after penile injection of "Jamaica oil" for the purpose of penile augmentation and discuss the utility of US and MRI in the management of this condition.

\section{Cases report}

\section{Case 1}

A 27-year-old male presented with penile swelling and painful erection. He denied any history of trauma but admitted to self-injection of "Jamaica oil" into the ventral aspect of his penis. On examination, the penile shaft was diffusely swollen with scar tissue especially on the ventral surface. Focal induration was felt at the dorsal surface of the penis. An US of the penis was done in the flaccid state using a 9-12 MHz linear array transducer. Diffuse subcutaneous swelling of the penile shaft was seen which showed increased echogenicity (fig 1). Mild diffuse hyperemia of this region was seen which was similar to that of the rest of the penile shaft. No collection was identified within the penile shaft and the corporal structures and glans penis were not involved. The patient was managed conservatively. 


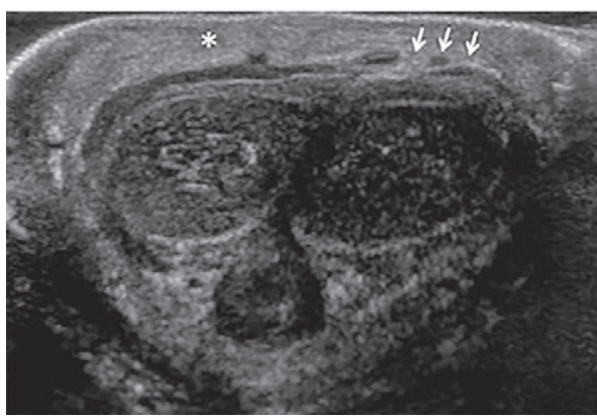

Fig 1. Transverse view of penile shaft shows diffuse thickening $\left({ }^{*}\right)$ of the subcutaneous tissue surround the Buck's fascia with increased echogenicity. Irregularity of the Buck's fascia is seen at the left side (arrows).

\section{Case 2}

A 20-year-old male presented with hardening of the penile shaft and painful erection. The patient had injected 80 milliliters of "Jamaica oil" thrice into the penile shaft 2 years ago. Four months prior to presentation at the clinic, he felt gradual hardening of the penile base, scrotum and suprapubic region. On examination, a superficial ulcer was seen at the ventral aspect of the penile shaft. An US scan of the penis showed a thick subcutaneous echogenic layer with posterior acoustic shadowing around the shaft with sparing of the glans penis. The echogenic areas extended through the Dartos fascia into the scrotal sac along the spermatic cords. There was also extension into the scrotal sac and superficially into the subcutaneous tissue of the lower abdominal wall (fig 2). There was no involvement of the corpora or fluid collection. Excision and bipedicled scrotal flap reconstruction of the denuded penile shaft was performed initially. Unfortunately the patient developed complications after the first procedure and eventually underwent debridement and degloving of penile shaft skin and skin grafting. Histology of the resected skin and soft tissue showed focal epidermal ulceration with underlying chronic inflammation. The dermis was widely replaced by numerous cystic spaces which were within a fibrotic/scarred dermis and widespread foreign body granulomatous response was seen.

\section{Case 3}

A 17-year-old patient presented with 3-day history of penile shaft swelling and pain. The patient gave a history of injecting "Jamaica oil" into his penile shaft 6 months ago. On examination, the penile shaft was swollen and erythematous which extended to the penile base and scrotum. A superficial ulcer was seen at the dorsum of the penile shaft. US of the penis was performed show-

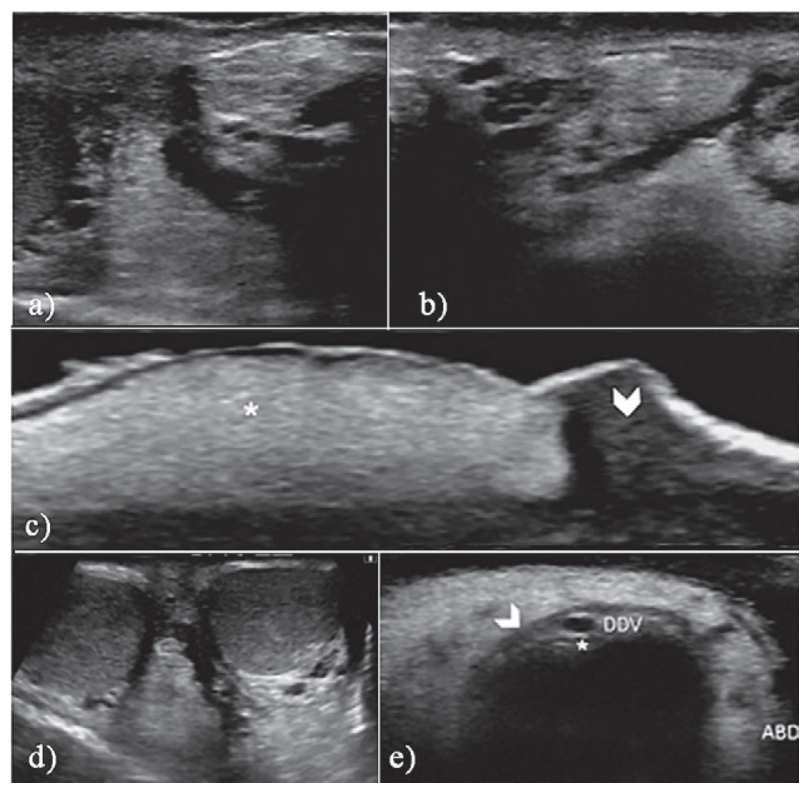

Fig 2. Transverse views of bilateral spermatic cords show echogenic soft tissue in keeping with foreign body reaction $(2 a, 2 b)$. Longitudinal view of the penile shaft shows echogenic material $(2 \mathrm{c}, *)$ within the subcutaneous layer of the penile shaft with sparing of glans penis (2c, arrowhead). Transverse views show echogenic material within the scrotal sac and anterior abdominal wall in keeping with foreign body extension (2d, 2e). Deep dorsal vein $(\mathrm{DDV})$ is seen $\left(2 \mathrm{e},{ }^{*}\right)$. No clear fat plane is seen around the Buck's fascia (2e, arrowhead).

ing diffuse increased echogenicity within the subcutaneous tissue around the penile shaft with extension to the anterior abdominal wall (fig 3a-d). No clear plane was seen between the echogenic foreign body and the Buck's fascia. The corporal structures and the glans penis were not involved. There was no abscess or deep extension of the superficial ulcer. MRI was subsequently performed with a $1.5 \mathrm{~T}$ machine. Multiplanar T1 and T2 weighted spin ECHO sequences revealed marked thickening of the subcutaneous tissue overlying the Buck's fascia, involving the shaft of penis and extending to the subcutaneous tissue of the suprapubic region. The thickened soft tissue showed hypointense T1 and T2 signal intensity suggestive of fibrosis (Fig 3e,f). The corpora cavernosa and corpus spongiosum showed normal signal. However, no definite plane was seen between the foreign body reaction and the Buck's fascia. MRI confirmed the absence of abscess. The patient subsequently underwent surgical debridement of the infected superficial ulcer. Intra-operatively the Buck's fascia was found to be scarred with no fat plane seen between the scar tissue and the fascia. On histological evaluation, the gross specimen showed a tan yellow firm fibrous appearance. Microscopy revealed 


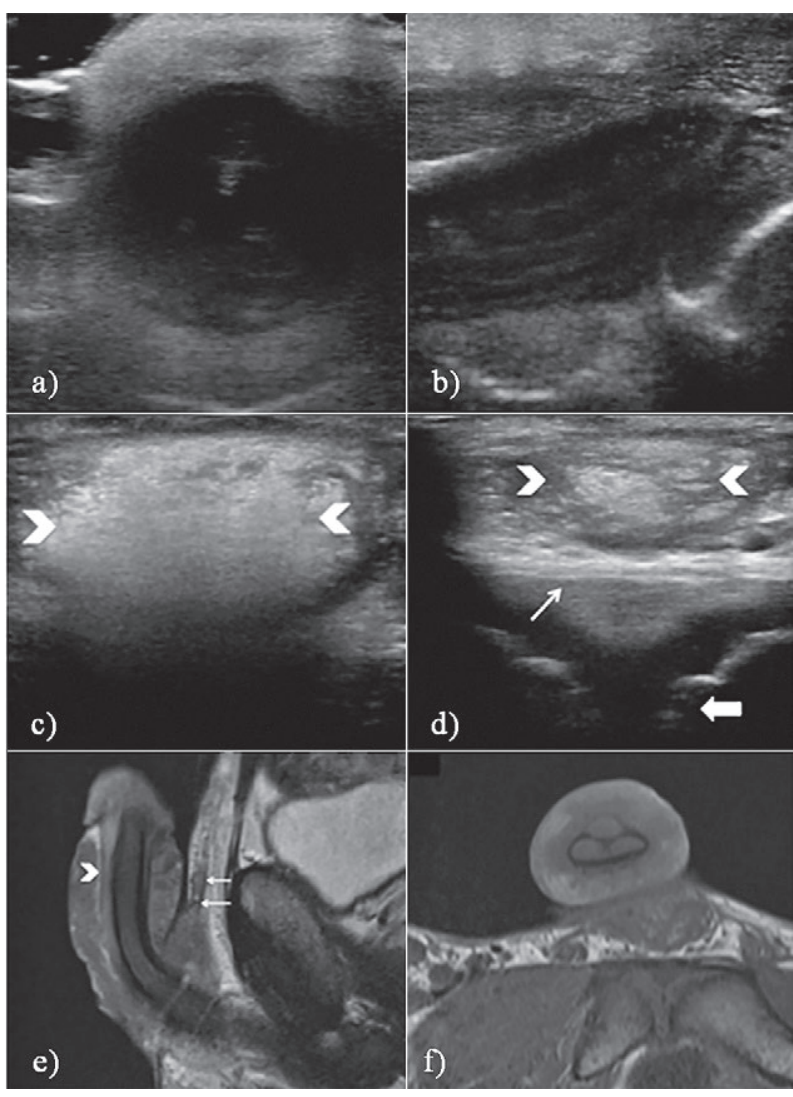

Fig 3. Transverse and longitudinal views show echogenic material around the penile shaft $(3 \mathrm{a}, 3 \mathrm{~b})$. Foreign body extension (arrowheads) is seen at the lower abdominal wall (3c) and at the level of the symphysis pubis ( $3 \mathrm{~d}$, solid arrow) anterior to the deep fascia ( $3 \mathrm{~d}$, thin arrow). MRI correlation shows hypo intense signal in sagittal T2-weighted and axial T1-weighted images within the subcutaneous layer of the penile shaft with extension to the lower anterior abdominal wall (3e, thin arrows) in keeping with fibrosis. Buck's fascia is seen (3e, arrowhead) to be intact. Axial T1 weighted sequence (f) shows hypointense signal in keeping with fibrosis.

many variably sized empty cystic spaces which were diffusely distributed throughout the dermis. Chronic inflammation and fibrosis was seen surrounding the cystic spaces with surrounding sheets of macrophages and foreign body-type multinucleated giant cells. The findings were consistent with sclerosing lipogranuloma.

\section{Case 4}

A 26-yr-old patient presented with 3-year history of penile swelling. He admitted to injecting "Jamaica oil" into the penile shaft and inserting 3 plastic bearings into the base of the penis 4 years ago. He has been having gradual penile swelling, skin tightening and progressive deformity. On examination induration was felt around

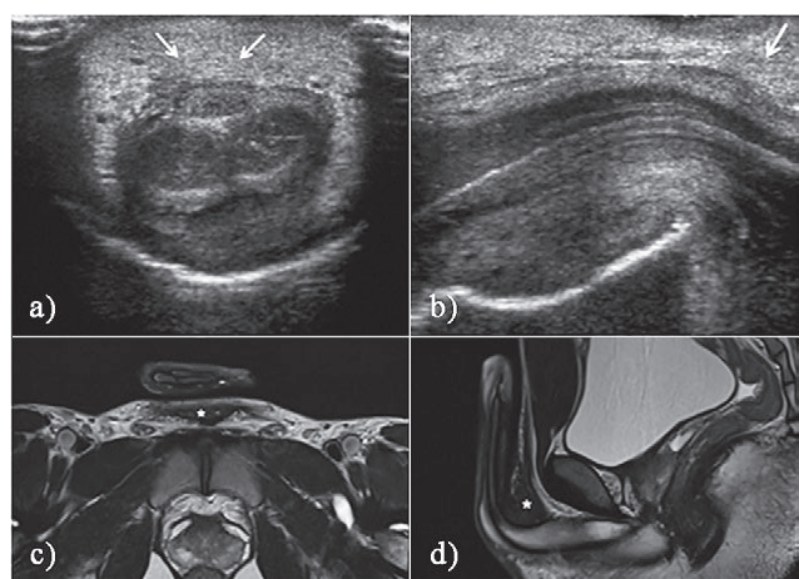

Fig 4. Diffuse increased echogenicity of the subcutaneous tissue is seen around the penile shaft extending to the penile base and anterior abdomen (4a,b; arrows). Corresponding MRI shows hypo intense T2 signal intensity $\left(4 \mathrm{c},{ }^{*}\right)$. The proximal extension to lower anterior abdomen is well demonstrated on the sagittal image $(4 \mathrm{~d}, *)$.

the penis, which extended to the root of the penis and anterior lower abdominal wall. US of the penis was performed showing diffuse thickening of the subcutaneous tissue around the penile shaft with increased echogenicity and posterior acoustic shadowing (fig $4 \mathrm{a}, \mathrm{b}$ ). The penile beads showed posterior acoustic shadowing. An MRI was performed on a $1.5 \mathrm{~T}$ machine revealed hypointense $\mathrm{T} 1$ and T2 signal in the subcutaneous layer around the Buck's fascia predominantly involving the ventral side, the appearance in keeping with fibrosis (fig 4c,d). The proximal extent of the fibrosis or foreign body reaction to the anterior abdominal wall at the pubic region was well demonstrated on the sagittal sequence. No focal abscess was detected.

\section{Discussions}

In Singapore, we have encountered patients performing penile self-augmentation with injection of "Jamaica Oil". There are many products available in this name, mostly advertised for hair growth, but the actual chemical composition is uncertain. Injection of mineral and other high viscosity oils into the penis results in various foreign body reactions with resultant scarring and deformity [1]. The potential complications include skin necrosis, phimosis, fistulation, abscess formation, erectile dysfunction and Fournier's gangrene [9-12]. The cause for foreign body reaction to injected oils is due to the body's inability to metabolize interstitial exogenous oils [3]. As a result the normal subcutaneous fat eventually becomes replaced by lakes of oil that can be seen interspersed with 
fibrous tissue and granulomatous chronic inflammatory reaction [2]. In most cases, the abnormality is localized to the penis but sometimes scrotal involvement and loco regional lymph node involvement can occur. The time of onset of granuloma formation has been reported from 2 days to 40 years post injection and most of the complications occur approximately after a year $[13,14]$.

US is the most commonly requested imaging modality in our institution for patients with foreign body injection because it is cheap, non-ionizing, readily available and offers real time assessment. Ultrasound is ideal in evaluating superficial structures like the penis in view of its high spatial resolution. The extent of dermal inflammation, presence or absence of collection and the depth of ulcers can also be evaluated with US. More importantly, US allow radiologists to determine if the plane between the indurated inflammatory tissue and the Buck's fascia is preserved which allows for complete surgical excision of affected tissue [15]. It is important for the surgeons to know if there is a clear place between the foreign body material and the Buck's fascia because for definitive treatment, complete excision of the foreign body is not possible if the Buck's fascia is involved and scarred [9]. Proximal extension of granulation tissue, scrotal involvement and loco regional lymph node involvement can also be evaluated.

The main drawback of US is that it is operator-dependent. It can also be difficult to scan these patients when the penis is deformed as in two of our patients where acoustic windows were limited due to the presence of superficial ulcers.

In all our patients, the main finding was that of thickening and increase echogenicity of the subcutaneous tissue surrounding the Buck's fascia. The corporal structures and glans penis were not involved in any of our patients, nor was there any abscess. This could be due to the Buck's fascia and fibrous layer of tunica albuginea acting as protective barriers. As a result the foreign body spreads along the subcutaneous plane to the base of the penis and into the anterior abdominal wall. In three of our patients, the foreign body reaction was seen involving the anterior abdominal wall. In one of our patients the imaging abnormalities extend through the Dartos fascia into the scrotum through the spermatic cords. Late cavernosal spread of foreign body has also been reported [16]. We postulate that even if a small amount of liquid foreign body entered the corpora cavernosa, the substance would be dispersed by the naturally occurring increased blood flow and sinusoidal spaces in this area, preventing it from eliciting a local response.

Although the US findings of penile paraffinomas has not been described in the literature, paraffinomas of breasts, buttocks, cervicofacial region and knees have been well documented [17-19] and show similar imaging finding to penile paraffinomas. In all these areas, when mineral oil or paraffin was injected, there is increased echogenicity of the soft tissues with posterior acoustic shadowing along fascial planes and involving adjacent structures. In three of our patients, the foreign body reaction spread to the lower anterior abdominal wall. The US findings at the lower anterior abdominal wall per se can provide diagnostic confusion if the appropriate history is not available. There has been a case report of petroleum jelly induced penile paraffinoma with inguinal lymphadenitis mimicking incarcerated inguinal hernia [20]. Also a case of breast paraffinoma in which the liquid paraffin spread along the fascial planes to involve the anterior abdominal wall was described and was misdiagnosed as liposarcoma [17].

In two of our patients we had MRI correlation that showed the proximal extent of foreign body reaction. Multiplanar capability of MRI offers many of the benefits of US which includes evaluating the extent of inflammation, involvement of Buck's fascia and presence of deep seated collections. MRI is also less operator dependent, and the anatomy is presented in a manner which is more easily understood by clinicians. The main indication for MRI is to evaluate the extension of the foreign body into the anterior abdomen. In such cases MRI helps the clinician to better visualize the proximal extent of foreign body reaction especially when surgery is planned. MRI is however, more expensive which is why it is often not the first imaging study requested.

For MRI evaluation of the penis our protocol includes T2 weighted sequences in axial, coronal and sagittal planes. T1 weighted sequences and TIRM sequence are also obtained in axial and sagittal planes. In both our patients, MRI showed corresponding low T1 and T2 signal intensity at the region of foreign body material/reaction consistent with scarring secondary to foreign body reaction. These imaging findings are also similar to paraffinomas in the breast [18]. Motoori et al [21] has reported the imaging findings in a case of sclerosing lipogranuloma of genitalia which showed moderately hyperintense T2 and isointense $\mathrm{T} 1$ signal intensities. The hypointense $\mathrm{T} 1$ and T2 signal intensities seen in our patients compared to those reported is likely due to the relatively late presentations and time of imaging in our patients.

Two of our patients were operated and the histology showed chronic inflammation and fibrosis surrounding cystic spaces and sheets of macrophages and foreign body-type multinucleated giant cells which correlates with the clinical and imaging diagnosis of foreign body reaction or sclerosing lipogranuloma. 
The recommended treatment option involves removal of foreign body material and granuloma followed by appropriate skin coverage $[1,22]$. To achieve definitive treatment, complete radical excision is necessary to remove the foreign body [23,24]. Successful treatment of granuloma with oral corticosteroids has also been reported [25].

In conclusion, we describe the US features of $4 \mathrm{pa}-$ tients and corresponding MRI appearance in 2 patients who injected "Jamaica oil" for penile augmentation. The imaging appearances of this condition are characteristic and show similarities to paraffinomas of other body parts. Early detection and complete excision is required in the case of penile paraffinoma to attain definitive anatomic and functional recovery.

Acknowledgment: We would like to thank $\mathrm{Dr} \mathrm{Ng}$ Fook Cheong ( Department of Urology, Changi General Hospital) and Dr Lee Song Liang Joshua ( Department of Urology, Changi General Hospital) for providing valuable clinical information and arranging for the scans.

\section{References}

1. Jeong JH, Shin HJ, Woo SH, Seul JH. A new repair technique for penile paraffinoma: bilateral scrotal flaps. Ann Plast Surg 1996; 37: 386-393.

2. Hohaus K, Bley B, Kostler E, Schonlebe J, Wollina U. Mineral oil granuloma of the penis. J Eur Acad Dermatol Venereol 2003; 17: 585-587.

3. Del Rosario RN, Barr RJ, Graham BS, Kaneshiro S. Exogenous and endogenous cutaneous anomalies and curiosities. Am J Dermatopathol 2005; 27: 259-267.

4. Lee T, Choi HR, Lee YT, Lee YH. Paraffinoma of the penis. Yonsei Med J 1994; 35: 344-348.

5. Bayraktar N, Başar İ. Penile paraffinoma. Case Rep Urol 2012; 2012: 202840.

6. Gurdal M, Karaman MI. An unusual case of penile augmentation: subcutaneous stone implantation. Urology 2002;59: 445.

7. Oh KJ, Park K, Kang TW, Kwon DD, Ryu SB. Subcutaneous metallic mercury injection for penile augmentation. Urology 2007; 69: 185e3-185e4.

8. Bjurlin MA, Carlsen J, Grevious M, et al. Mineral Oil-induced Sclerosing Lipogranuloma of the Penis. J ClinAesthet Dermatol 2010; 3: 41-44.

9. Moon DG, Yoo JW, Bae JH, Han CS, Kim YK, Kim JJ. Sexual function and psychological characteristics of penile paraffinoma. Asian J Androl 2003; 5: 191-194.
10. Lee SW, Bang CY, Kim JH. Penoscrotal reconstruction using groin and bilateral superomedial thigh flaps: a case of penile vaselinoma causing Fournier's gangrene. Yonsei Med J 2007; 48: 723-726.

11. Steffens J, Kosharskyy B, Hiebl R, Schonberger B, Rottger $\mathrm{P}$, Loening S. Paraffinoma of the external genitalia after autoinjection of vaseline. Eur Urol 2000; 38: 778-781.

12. Cohen JL, Keoleian CM, Krull EA. Penile paraffinoma: self-injection with mineral oil. J Am Acad Dermatol 2002; 47(5 suppl): S251-S253.

13. Eandi JA, Yao AP, Javidan J. Penile paraffinoma: the delayed presentation. Int Urol Nephrol 2007; 39: 553-555.

14. Pehlivanov G, Kavaklieva S, Kazandjieva J, Kapnilov D, Tsankov N. Foreign-body granuloma of the penis in sexually active individuals (penile paraffinoma). J Eur Acad Dermatol Venereol 2008; 22: 845-851.

15. O'Rourke MG. Lipogranulomata of male genitalia. Br J Urol 1967;39:426-430.

16. Karakan T, Ersoy E, Hasçiçek M, Özgür BC, Özcan S, Aydin A. Injection of Vaseline under Penis Skin for the Purpose of Penis Augmentation. Case Rep Urol 2012; 2012: 510612.

17. Wong KT, Lee PS, Chan YL, Chow LT. Paraffinoma in anterior abdominal wall mimicking liposarcoma. Br J Urol 2003;76:264-267.

18. Erguvan-Dogan B, Yang WT. Direct injection of paraffin into the breast: mammographic, sonographic, and MRI features of early complications. AJR Am J Roentgenol 2006; 186: 888-894.

19. Gu DH, Yoon DY, Chang SK, et al. CT features of foreign body granulomas after cosmetic paraffin injection into the cervicofacial area. Diagn Interv Radiol 2010; 16: 125-128.

20. Sejben I, Racz A, Svebis M, Patyi M, Cserni G. Petroleum jelly-induced penile paraffinoma with inguinal lymphadenitis mimicking incarcerated inguinal hernia. Can Urol Assoc J 2012; 6: E137-E139.

21. Motoori K, Takano H, Ueda T, Ishihara M. Sclerosing lipogranuloma of male genitalia: CT and MR images. $J$ Comput Assist Tomogr 2002; 26: 138-140.

22. Santucci RA, Zehring RD, McClure D. Petroleum jelly lipogranuloma of the penis treated with excision and native skin coverage. Urology 2000; 56: 331.

23. Rosenberg E, Romanowsky I, Asali M, Kaneti J. Three cases of penile paraffinoma: a conservative approach. Urology 2007; 70: 372e9-372e10.

24. Al-Ansari AA, Shamsodini A, Talib RA, Gul T, Shokeir AA. Subcutaneous cod liver oil injection for penile augmentation: review of literature and report of eight cases. Urology 2010; 75: 1181-1184.

25. Lawrentschuk N, Angus D, Bolton DM. Sclerosing lipogranuloma of the genitalia treated with corticosteroids. Int Urol Nephrol 2006; 38: 97-99. 